\title{
Simulation of diopter changes in radio frequency conductive keratoplasty in the cornea
}

\author{
J. Pearce ${ }^{1}$, D. Panescu ${ }^{2}$ \& S. Thomsen ${ }^{3}$ \\ ${ }^{I}$ Department of Electrical \& Computer Engineering, \\ The University of Texas at Austin, U.S.A. \\ ${ }^{2}$ Refractec Inc., Irvine CA, U.S.A. \\ ${ }^{3}$ Department of Biomedical Engineering, \\ The University of Texas at Austin, U.S.A.
}

\begin{abstract}
Correction of hyperopia (far-sightedness) is accomplished by decreasing the radius of curvature of the cornea. Radio frequency (RF) current at $350 \mathrm{kHz}$ is presently being used to the required correction. The procedure is termed Conductive Keratoplasty and temperature increases induced by the RF current shrink corneal collagen. Finite difference method (FDM) numerical models of the electric and current density fields, thermal fields, and resulting damage and collagen shrinkage fields have been used to study the effects of electrode voltage and power on transient tissue impedance and diopter change obtained from this process. Water is by far the most thermodynamically active tissue constituent, and must be taken into account in order to obtain realistic results. Thermal damage processes are highly non linear, typically following Arrhenius kinetic rate-limited process formulations, or their equivalent. The FDM model calculations predict water loss and thermal damage in the form of loss of birefringence in corneal collagen, which can be directly compared to histologic section using Transmission Polarizing Microscopy. Numerical model results compare well with experimental measurements and have been used to study the relative importance of the governing physical phenomena and the feasibility of constant power RF activation.
\end{abstract}

Keywords: surgical simulation, cornea, hyperopia correction, radio frequency surgery, needle electrode, numerical models, thermal damage prediction. 


\section{Introduction}

Hyperopia (far-sightedness), caused by inadequate curvature of the cornea, can be corrected by thermally shrinking corneal collagen. Radio frequency (RF) current delivered through a monopolar electrode inserted into the cornea to shrink corneal collagen has recently been introduced as a clinically viable option [1]. Typical RF activations are at a frequency of $350 \mathrm{kHz}$, are $0.6 \mathrm{~s}$ long consist of exponentially damped constant voltage pulses delivered at a nominal rate of $7.5 \mathrm{kHz}$. The active electrode is $90 \mu \mathrm{m}$ in diameter and $450 \mu \mathrm{m}$ long with a "trocar" bevelled tip. The electrode is inserted into the cornea at 8 peripheral points on a 3, 3.5 or $4 \mathrm{~mm}$ radius, (i.e. 8, 16 or 24 points), as necessary, around the cornea to achieve the desired correction. A teflon electrode cap $0.2 \mathrm{~mm}$ in radius prevents over insertion of the electrode into the cornea. The return electrode comprises both legs of the speculum used to retract the eyelids during use.

Finite difference method (FDM) numerical models in axisymmetric cylindrical coordinates ( $r, \mathrm{z}$ ) have been applied to study the RF fields, the temperature fields and resulting collagen shrinkage. The Finite Difference Method was chosen since the model space closely follows the coordinate system and it will yield useful results with minimum development time. Commercial Finite Element Method packages are currently not capable of including the tissue damage models so necessary to proper study of the process. The models have been applied to study the relative importance of tissue water the time evolution of tissue resistance and resulting collagen shrinkage obtained from treatments. The predicted acute change in corneal diopter value is the standard of comparison among the models.

\section{Methods}

The electromagnetic numerical model consists of Finite Difference Method (in control volume form) solution of the quasi-static Laplace equation:

$$
\nabla \bullet(\sigma \nabla \mathrm{V})=0
$$

where: $\mathrm{V}=$ potential $(\mathrm{V})$ and $\sigma=$ electrical conductivity $(\mathrm{S} / \mathrm{m})$. The model space was 101 radial and 51 vertical control volumes in the tissue. The electric field model solves the inhomogeneous form of the Laplace equation. The boundary conditions are: fixed potential at the electrode surface and $\mathrm{R}_{\max }$, and zero normal flux at the air surface and in the aqueous humour. The process is sufficiently symmetrical near to the electrode that an axisymmetric model space is a good representation of the overall geometry. The model equations were derived from the integral form of continuity and allow each FDM control volume to have unique electrical and thermal properties that change in accordance with changes in tissue temperature and water volume fraction during the activation. In order to provide adequate spatial resolution and an acceptable representation of the electrode geometry the maximum outer radius, $R_{\max }$ is limited to about 1 to $2 \mathrm{~mm}$ as a practical matter. The speculum return electrodes are 1 to $2 \mathrm{~cm}$ away from the 
lesion site, and behave, in effect, like flat plates. In the truncated FDM model space the speculum electrode was replaced by a coaxial cylinder of fixed potential at $\mathrm{R}_{\max }$. The fixed potential and $\mathrm{R}_{\max }$ were chosen to obtain an accurate representation of the electrode shape and an equivalent location for the ground cylinder (outside the model space) at dimensions similar to the location of the speculum return electrodes in vivo.

Special care was taken in the FDM formulation to accurately represent the electrode tip. The tip is sharp and has a singularity in the E-field at the centerline. The sharpened trocar shape was modeled as approximately a cone. Special FDM equations were used in the cone region so that the resulting E-field would be normal to the electrode-tissue interface and the "stair-step" characteristics typical of FDM models could be avoided.

The thermal model is a transient solution of the energy balance driven by the $\mathrm{RF}$ volume power density field $\left(\mathrm{Q}_{\mathrm{gen}}\right)$ and includes conduction heat in the tissue, electrode, and teflon electrode shoulder used to limit electrode penetration depth as well as water loss phenomena (2).

$$
\rho c \frac{\partial T}{\partial t}=-\nabla \bullet(k \nabla T)+Q_{\text {gen }}-h_{f g} \frac{\partial m}{\partial t}-\text { Losses }
$$

where: $\rho=$ density $\left(\mathrm{kg} / \mathrm{m}^{3}\right), \mathrm{c}=$ specific heat $(\mathrm{J} / \mathrm{kg}-\mathrm{K}), \mathrm{T}=$ temperature $(\mathrm{K}), \mathrm{k}=$ thermal conductivity $(\mathrm{W} / \mathrm{m}-\mathrm{K}), \mathrm{Q}_{\text {gen }}=\mathrm{E}$-field power density $\left(\mathrm{W} / \mathrm{m}^{3}\right), \mathrm{h}_{\mathrm{fg}}=$ phase change enthalpy $(\mathrm{J} / \mathrm{kg})$, and $\partial \mathrm{m}=$ water mass volume density changing phase $\left(\mathrm{kg} / \mathrm{m}^{3}\right)$. The thermal model includes 21 control volumes above the tissue surface to represent the electrode and surrounding teflon shoulder.

Tissue water is by far the most thermodynamically active constituent. In addition to the energy balance contribution, the volume fraction of water determines the electrical conductivity, density, thermal conductivity and specific heat of the tissues. Tissue water vaporization was considered to be equilibrium boiling at $100{ }^{\circ} \mathrm{C}$ and atmospheric pressure. During activation as the temperature rises, and/or as the tissue dries out, tissue electrical and thermal properties are changed accordingly and the electric and RF power density fields recalculated. Convection, radiation and evaporation boundary conditions are specified at the exposed surfaces, as appropriate ("Losses") with evaporation modeled by experimental correlations [2], though evaporation is not an important contribution in these short activations. Thermal contact resistances were included between the electrode and tissue and the electrode and Teflon shoulder.

The electrical conductivity is [2]:

$$
\sigma(\mathrm{T}, \mathrm{w})=\sigma(0) \mathrm{w} \mathrm{e}^{0.015\left[\mathrm{~T}-\mathrm{T}_{0}\right]}
$$

where: $\sigma(0)$ is the electrical conductivity of the corneal electrolyte at initial temperature $\mathrm{T}_{0}, \mathrm{w}$ is the volume fraction of tissue water and a temperature dependence of $1.5 \%$ per ${ }^{\circ} \mathrm{C}$ has been assumed, as is typical for electrolytes. 
Thermal properties also change with temperature and water fraction in the model. Values were determined from published correlations for water and protein content $[2,3]$ :

$$
\begin{gathered}
\rho=\frac{1}{\mathrm{~m}_{\mathrm{w}}^{*}+0.649 \mathrm{~m}_{\mathrm{p}}^{*}+1.227 \mathrm{~m}_{\mathrm{f}}^{*}} \\
\mathrm{c}=4.2 \mathrm{~m}_{\mathrm{w}}^{*}+1.09 \mathrm{~m}_{\mathrm{p}}^{*}+2.3 \mathrm{~m}_{\mathrm{f}}^{*} \\
\mathrm{k}=\rho\left[6.28 \mathrm{~m}_{\mathrm{w}}^{*}+1.17 \mathrm{~m}_{\mathrm{p}}^{*}+2.31 \mathrm{~m}_{\mathrm{f}}^{*}\right]
\end{gathered}
$$

where: $\rho=$ density $\left(\mathrm{kg} / \mathrm{m}^{3}\right), \mathrm{c}=$ specific heat $(\mathrm{J} / \mathrm{kg}-\mathrm{K}), \mathrm{k}=$ thermal conductivity $(\mathrm{W} / \mathrm{m}-\mathrm{K})$, and $\mathrm{m}_{\mathrm{i}}^{*}=$ the mass fractions of water, protein and fat, respectively. Cornea consists of collagenous proteins and water, and relatively few chondrocytes with epithelial cells on both surfaces.

The damage processes were included as first order kinetic models. In general first order kinetic models take the form:

$$
\ln \left\{\frac{\mathrm{C}(0)}{\mathrm{C}(\tau)}\right\}=\int_{0}^{\tau} \mathrm{A} \mathrm{e}^{-\frac{\mathrm{E}}{\mathrm{RT}}} \mathrm{dt}
$$

where: $\mathrm{C}$ is the remaining concentration of undamaged protein or other measurable quantity, A is the "frequency factor" $\left(\mathrm{s}^{-1}\right)$ a measure of collision frequency, $\mathrm{E}$ is the activation energy $(\mathrm{J} / \mathrm{mole}), \mathrm{R}$ the gas constant $(8.314 \mathrm{~J} / \mathrm{mole}$ $\mathrm{K})$, and $\mathrm{T}$ the temperature $(\mathrm{K})$. For birefringence loss $[4]\left(\mathrm{A}=1.61 \times 10^{45} \mathrm{~s}^{-1}\right.$ and $\mathrm{E}=306 \mathrm{~kJ} / \mathrm{mole})$, and for epithelial cell damage skin coefficients were used $(\mathrm{A}=$ $299 \times 10^{96} \mathrm{~s}^{-1}$ and $\left.\mathrm{E}=628 \mathrm{~kJ} / \mathrm{mole}\right)$.

The model for collagen shrinkage $[5,6]$ has a different functional form, though similar to the Arrhenius formulation above. Its use in the calculations has been described elsewhere [7,8]. The practical maximum for shrinkage in length is about $65 \%$. Above that value the collagen gellifies and loses its strength. The model functions utilize a non-dimensional time axis, $t / \tau_{2}$ to estimate the shrinkage. The functional form of $\tau_{2}$ contains the kinetic nature of the process, but in the form of an exposure time rather than a rate of formation, as in (5). To use the model at each point in space and time an equivalent value for the increment in $t / \tau_{2}$ is calculated and accumulated until shrinkage is calculated.

The corneal radius of curvature, expressed in diopter form, D, is [9]:

$$
D=(n-1) / R
$$

where: $\mathrm{D}=$ diopters, $\mathrm{n}=$ index of refraction of the cornea and $\mathrm{R}=$ radius of the cornea $(\mathrm{m})$. For cornea with a nominal index of refraction of 1.336 and radius of 
curvature of $8 \mathrm{~mm}$, the diopter value is 42 . The result of shrinkage at the points of RF activation is reduction in the circumference of the cornea and its attendant radius, at constant corneal surface area. To calculate the diopter value after shrinkage requires iterative solution of a transcendental relation. It turns out, somewhat surprisingly, that the diopter value is linear with cornea circumference shrinkage (Figure 1).

\section{Results}

The radial increment, dr, was fixed at $0.0082 \mathrm{~mm}$ and the vertical increment, $\mathrm{dz}$, was $0.028 \mathrm{~mm}$ in tissue and $0.025 \mathrm{~mm}$ above the tissue. The model tissue space was $0.8 \mathrm{~mm}$ thick (cornea thickness $=0.7 \mathrm{~mm}$ with $0.1 \mathrm{~mm}$ of aqueous humour) and the electrode and teflon shoulder extended $0.5 \mathrm{~mm}$ above the cornea surface. The model radius was $0.82 \mathrm{~mm}$, and the boundary voltage was set to obtain an equivalent ground cylinder located at $15 \mathrm{~mm}$ radius. The electric field solution typically required 2,000 to 3,000 iterations to converge adequately (total electrode current change less than $0.05 \%$ in 100 iterations).

\subsection{Standard constant voltage model}

A standard model was selected in the mid-range of typical Conductive Keratoplasty treatments to serve as a basis of comparison for the parametric variation study. The exponential RF pulse maximum voltage was $70.7 \mathrm{~V}(\mathrm{rms})$, time constant $15 \mu$ s at a pulse repetition rate of $7.46 \mathrm{kHz}$ (duty cycle $22 \%$ ). In all of the models the reference ground cylinder was at a radius of $15 \mathrm{~mm}$, simulating the position of the eyelid speculum electrode.

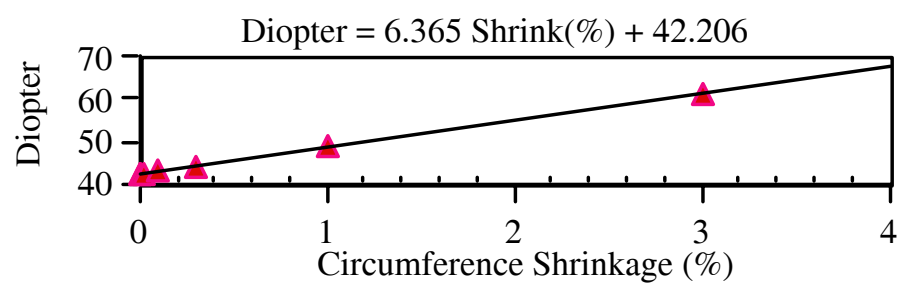

Figure 1: $\quad$ Plot of corneal diopter value $v s$ circumferential shrinkage.

The eight RF spots were placed on a circumference of radius $3.5 \mathrm{~mm}$ in the model calculation, and the original radius of curvature of the cornea was assumed to be $8 \mathrm{~mm}$. The cornea electrical conductivity was $1.125 \mathrm{~S} / \mathrm{m}$ (in the mid-range of published values) and initial electrode resistance was $1422 \Omega$, typical of a clinical treatment (initial time average power $=0.197 \mathrm{~W}$, final power $=0.305 \mathrm{~W})$. The total RF activation time was $0.6 \mathrm{~s}(\sim 4,500 \mathrm{RF}$ pulses $)$ and the cooling time was $0.4 \mathrm{~s}$, for a total model time of $1 \mathrm{~s}$. Electrode thermal 
properties (stainless steel) and the fine spatial resolution meant that a time step of $1.5 \mu \mathrm{s}$ was used in the transient thermal model.

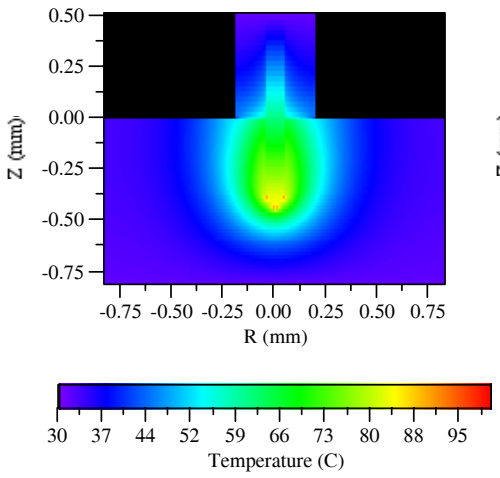

(a)

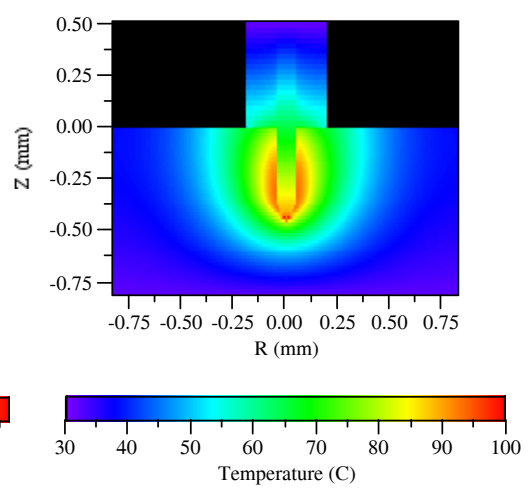

(b)

Figure 2: Temperature field, $\mathrm{T}(\mathrm{r}, \mathrm{z})$ : (a) at $\mathrm{t}=0.3 \mathrm{~s}$ and (b) at end of $\mathrm{RF}$ activation, $\mathrm{t}=0.6 \mathrm{~s}$ (both scales $30^{\circ}$ to $100^{\circ} \mathrm{C}$ ).

The electric field and thermal properties were recalculated when the tissue within 2 electrode radii of the tip increased $10 \mathrm{C}$ or at least every $25 \mathrm{~ms}$ (24 times during RF activation for this model). The temperature dependence of electrical conductivity accounts for the increase in time average power from the initial to final values as the electrode voltage was held constant.

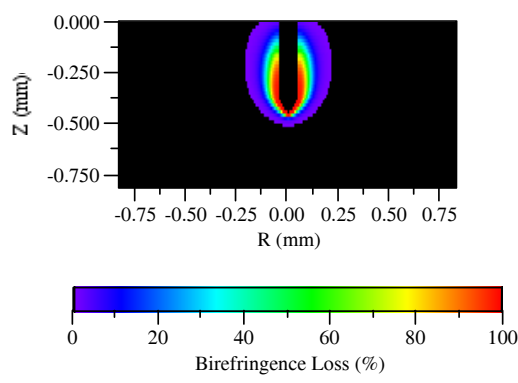

(a)

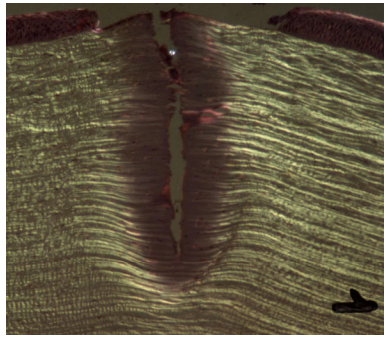

(b)

Figure 3: Damage field at $t=1 \mathrm{~s}$. (a) birefringence loss from model $(10 \%$ and $90 \%$ loss contours) and (b) a close-up TPM view of the histologic section, original magnification $25 \mathrm{X}(\mathrm{bar}=0.1 \mathrm{~mm})$.

Tissue water dominates the development of the lesion. The initial RF pulses cause substantial boiling at the electrode tip where the impedance rises, shifting 
the bulk of the power dissipation up the electrode shaft toward the cornea surface. This effect can clearly be seen in Figure 2(b) where the tip-weighted heating seen in Figure 2(a) the bulk of the late term heating is closer to the tissue surface. Heat transfer to the electrode and its teflon shoulder are important to the final result. The model predicts significant tissue water loss only in the immediate vicinity of the electrode tip.

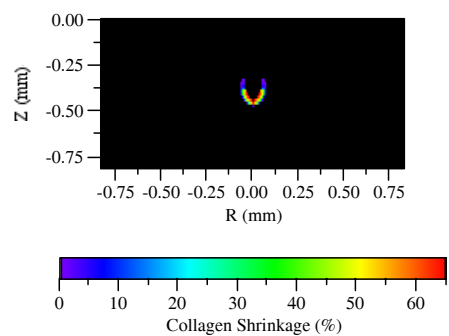

(a)
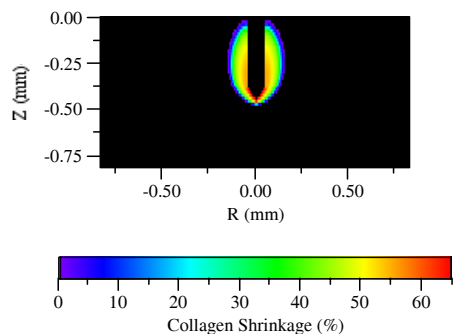

(b)

Figure 4: $\quad$ Collagen shrinkage (0 to $65 \%$ ) at (a) $0.3 \mathrm{~s}$ and (b) $1.0 \mathrm{~s}$.

Figure 3(a) is the predicted collagen birefringence loss boundary. Figure 3(b) is a Transmission Polarizing Microscopy (TPM) view of a typical birefringence loss measurement in pig cornea (original magnification $25 \mathrm{X}$, bar $=0.1 \mathrm{~mm}$ ). The relative scale of the images can be obtained from the relative lengths of the electrode $(450 \mu \mathrm{m})$. The predicted boundaries compare quite favorably with the histologic result. Figure 4 shows the predicted shrinkage at $0.3 \mathrm{z}$ and $1 \mathrm{~s}$ (corresponding to the temperature fields in Figure 2)

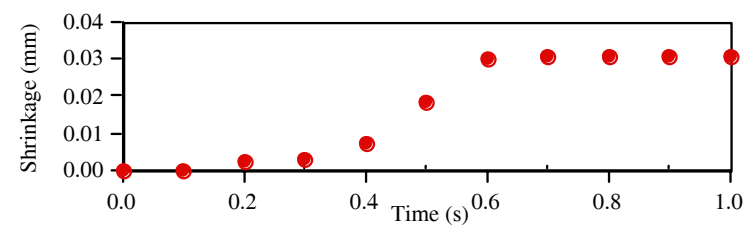

Figure 5: Time evolution of collagen shrinkage ( 0 to $2.4 \%$ of total length).

In Fig. 5 the transient collagen shrinkage plot shows that most of the change in length occurs in the last $100 \mathrm{~ms}$ of RF heating as the temperature profile advances toward the surface. In that plot, a length change of $-0.03 \mathrm{~mm}$ corresponds to a change in circumference of $-2.4 \%$, which is the origin of the diopter change from 42 to 52 for this numerical model. Again, significant postoperative relaxation in collagen shrinkage would be expected.

\subsection{Study of the effects of constant power RF activation}

We have already reported on the effects of uncertainty in tissue impedance and electrode voltage [8] in constant voltage experiments. In brief, a $10 \%$ 
uncertainty in initial electrode impedance (as reflected by uncertainty in the conductivity of the cornea rather than differences in the return electrode geometry) results in predicted acute diopter values between 47 and 58, a substantial variation around the central value of 52. We have subsequently studied the effect of using a constant power activation, rather than constant voltage.

Review of the analytical solutions for the axisymmetric coaxial line and hemispherical cap electric fields suggests that both the geometry of the power density field, $\left(\mathrm{Q}_{\mathrm{gen}}=\sigma|\mathrm{E}|^{2}\right)$, and adiabatic heating rate at the electrode surface are insensitive to the electrical conductivity when the electrode total power is held constant. Consequently, we would expect that a constant power RF delivery system would be less sensitive to uncertainty in electrical conductivity. However, this turns out not to be the case.

For this model series the same uncertainty in tissue electrical conductivity was used: $\sigma_{0}=1.013$ to $1.25 \mathrm{~S} / \mathrm{m}$ (the standard model above had $\sigma_{0}=1.125$ $\mathrm{S} / \mathrm{m})$. The approximate constant power equivalent RF activation was obtained with a continuous sine wave at time average power $\mathrm{P}=0.27 \mathrm{~W}$ for $0.6 \mathrm{~s}(2.2 \%$ shrinkage at this setting compared to $2.4 \%$ from Figure 5). The initial electrical conductivity was varied from 1.013 to $1.25 \mathrm{~S} / \mathrm{m}$ at a constant electrode time average power of $0.27 \mathrm{~W}$ applied for $0.6 \mathrm{~s}$ with $0.4 \mathrm{~s}$ allowed for cooling. The lower resistance case, at $1.25 \mathrm{~S} / \mathrm{m}$ (initial resistance $\mathrm{R}_{0}=1507 \Omega$ ) is virtually identical to the comparison case at $1.125 \mathrm{~S} / \mathrm{m}$ (initial resistance $\mathrm{R}_{0}=1667 \Omega$ ) the final diopter value $\mathrm{D}=57$ for both models. However, the lower value of electrical conductivity, $1.013 \mathrm{~S} / \mathrm{m}\left(\mathrm{R}_{0}=2100 \Omega\right)$, resulted in a significantly larger final diopter value, $\mathrm{D}=62$. In this case, the numerical model shows the same trend as the in vitro and in vivo experiments. Experience teaches that when the electrical conductivity is lower than the nominal value the power must be decreased to obtain a satisfactory result.

The reason for this trend is not clear. However, it is important to bear in mind that the actual electric field is neither approximately hemispherical nor approximately cylindrical near the tip of the electrode and also that the irreversible tissue alteration processes are highly non linear. Besides the hyperbolic/exponential nature of the damage kinetics (equation 5) collagen shrinkage saturates at about 60 to $65 \%$. Moreover, small differences in evolution of water vapor around the tip can have a larger effect on the final result, as it can drive the dominant heating zone up the electrode shaft earlier in the RF activation.

\section{Discussion}

The numerical model includes all of the relevant physical phenomena in Conductive Keratoplasty. The present work represents a substantial improvement in modeling of the CK process since it focuses non alterations in the tissue rather than temperature alone [10]. The model is sufficiently accurate 
that predictions of corneal shrinkage obtained can be used for understanding experimental results, treatment planning, and evaluation of treatment protocols. The models indicate that the initial shrinkage occurs at the tip of the electrode and heating and shrinkage gradually move up the electrode shaft during the later stages of RF activation. The result is that while the initial heating is highest at the electrode tip, it is not highest there for the entire activation.

We note that it is essential to include tissue water loss in any realistic model, both for the energy required and for the effect of water loss on the physical properties of the tissue. There is, for example, no other mechanism that will describe the increase in impedance commonly observed experimentally at the end of the RF activation when cornea is heated at the high end of the therapeutic range. Boiling at the electrode tip begins within the first few RF pulses, and proceeds to alter the distribution of current and power density.

\section{References}

[1] Refractec, Inc. Irvine CA, 2001.

[2] Pearce J.A. and Thomsen S.; The effect of vessel architecture on fusion by radio frequency current; Proc. SPIE 3249, pp. 217-228, 1998.

[3] Diller K.R., Valvano J.W. and Pearce J.A.; "Bioheat Transfer"; Chapter 4, section 4 in The CRC Handbook of Thermal Engineering, pp. 4-114 - 4187, F. Kreith, Ed., CRC Press, Boca Raton, FL, 2000. Original data for correlations excerpted from: T.E. Cooper and G.J. Trezek; Correlation of thermal properties of some human tissues with water content; Aerosp. Med., 42, pp. 24-27, 1971.

[4] Pearce J.A., Thomsen S., Vijverberg H. and McMurray T.; Kinetics for birefringence changes in thermally coagulated rat skin collagen, Proc. SPIE vol.1876, pp. 180-186, 1993.

[5] Chen S.S., Wright N.T. and Humphrey J.D.; Heat-induced changes in the mechanics of a collagenous tissue: isothermal, isotonic shrinkage, Trans. A.S.M.E. J. Biomech. Engr., 120, pp. 382-388, 1998.

[6] Chen S.S., Wright N.T. and Humphrey J.D.; Phenomenological evolution equations for heat-induced shrinkage of a collagenous tissue, I.E.E.E. Trans. on BME, vol. 45, pp. 1234-1240, 1998.

[7] Pearce JA. Corneal Reshaping by Radio frequency current: numerical model studies, Proc. SPIE, v.4247, pp109-118, January 2001.

[8] Pearce JA. and Panescu D. Radio frequency conductive keratoplasty in the cornea: prediction of diopter changes in numerical models. (invited paper) Proc. IEEE-Engr. in Med. \& Biol. Soc. 26th Annual Meeting, pp5426-5429, September 2004.

[9] Wysecki G. and Stiles W.S., Color Science: Concepts and Methods, Quantitative Data and Formulas. New York, John Wiley, 1967.

[10] Enrique J Berjano E.J., Jorge L Ali J.L. and Javier Saiz J. Modeling for radio-frequency conductive keratoplasty: implications for the maximum temperature reached in the cornea. Physiol. Meas. 26 157-172, 2005. 